\title{
Conhecimento da puérpera sobre amamentação na Atenção Básica
}

\author{
Knowledge of puerpera on breastfeeding in Basic Attention \\ Conocimiento de la puérpera sobre lactancia en la Atención Básica
}

\begin{abstract}
Danielle Crisóstomo Guimarães ${ }^{1}$, Barbara Nepomuceno Lima ${ }^{1}$, Agna Soares da Silva Menezes ${ }^{1,2}$, Cláudia Moreira Gomes ${ }^{2}$, Priscila Vieira Borges ${ }^{2}$, Nayara Teixeira Gomes ${ }^{3}$, Gracielle Soares da Silva Ruas $^{4}$.
\end{abstract}

\section{RESUMO}

Objetivo: Avaliar o conhecimento da puérpera sobre a importância do aleitamento materno na Atenção Primária no município de Montes Claros - MG . Metodologia: Pesquisa tansversal com abordagem qualitativa realizada com 12 puérperas de até 42 dias pós-parto, cadastradas e acompanhadas nas equipes de Saúde da Família, Montes Claros - MG. Resultados: As puérperas identificam os benefícios do aleitamento materno na prevenção de doenças e para o desenvolvimento da criança, além de reconhecerem a superioridade e praticidade da amamentação em comparação a oferta de fórmulas industrializadas. Dificuldades relacionadas às mamas caracterizaram a principal dificuldade com a técnica da mamada entre as entrevistadas. Algumas puérperas relataram não ter recebido informações sobre o assunto durante o pré-natal e puerpério. Conclusão: Percebese um conhecimento prévio das puérperas sobre o aleitamento, mas o tema necessita ser mais abordado pelos profissionais no acompanhamento ao pré-natal e puerpério. Além da oferta de ações de educação em saúde para esclarecimento de dúvidas e mitos sobre a amamentação e assim contribuir para diminuição do índice de desmame precoce.

Palavra chave: Atenção Primária à saúde, Aleitamento Materno, Período Pós-Parto, Desmame.

\begin{abstract}
Objective: To evaluate the knowledge of the puerpera about the importance of breastfeeding in Primary Care in the municipality of Montes Claros - MG. Methodology: Tansversal research with a qualitative approach performed with 12 puerperae of up to 42 days postpartum, enrolled and accompanied in the Family Health teams, Montes Claros - MG. Results: The mothers identify the benefits of breastfeeding in the prevention of diseases and in the development of the child, besides recognizing the superiority and practicality of breastfeeding in comparison to the offer of industrialized formulas. Difficulties related to breasts characterized the main difficulty with the technique of breastfeeding among the interviewees. Some puerperae reported not having received information on the subject during the prenatal and puerperium. Conclusion: A prior knowledge of puerperal women about breastfeeding is perceived, but the subject needs to be more approached by professionals in the prenatal and puerperal follow-up. In addition to offering health education actions to clarify doubts and myths about breastfeeding and thus contribute to a decrease in the rate of early weaning.
\end{abstract}

Keyword: Primary Health Care, Breastfeeding, Postpartum, Weaning.

${ }^{1}$ Faculdades Unidas do norte de Minas (FUNORTE), Montes Claros-MG.

2Universidade Estadual de Montes Claros-MG (UNIMONTES). E-mail: cllaugomes@yahoo.com.br

${ }^{3}$ Secretaria Municipal de Saúde de Montes Claros-MG

${ }^{4}$ Academia Top Gym, Montes Claros-MG. 


\section{RESUMEN}

Objetivo: Evaluar el conocimiento de la puérpera sobre la importancia de la lactancia materna en la Atención Primaria en el municipio de Montes Claros - MG. Metodología: Investigación tansversal con abordaje cualitativo realizada con 12 puérperas de hasta 42 días posparto, catastradas y acompañadas en los equipos de Salud de la Familia, Montes Claros - MG. Resultados: Las personas que han dado a luz identifican los beneficios de la lactancia materna en la prevención de enfermedades y el desarrollo del niño, además de reconocer la superioridad y la practicidad de la lactancia en comparación con la oferta de fórmulas industrializadas. Dificultades relacionadas con las mamas caracterizaron la principal dificultad con la técnica de la mamada entre las entrevistadas. Algunas puérperas relataron no haber recibido informaciones sobre el tema durante el prenatal y puerperio. Conclusión: Se percibe un conocimiento previo de las puérperas sobre la lactancia, pero el tema necesita ser más abordado por los profesionales en el seguimiento al prenatal y puerperio. Además de la oferta de acciones de educación en salud para aclarar dudas y mitos sobre la lactancia materna y así contribuir a la disminución del índice de destete precoz.

Palabra Claves: Atención Primaria a la salud, Lactancia Materna, Período Post-Parto, Desmame.

\section{INTRODUÇÃO}

O período do puerpério é definido como o tempo de seis a oito semanas após o parto, podendo ser dividido em três períodos : imediato $\left(1^{\circ}\right.$ ao $\left.10^{\circ} \mathrm{dia}\right)$, tardio $\left(11^{\circ} \mathrm{ao} 42^{\circ} \mathrm{dia}\right)$ e remoto (a partir de $\left.45^{\circ}\right)$. O período puerperal é de extrema importância na vida da mulher, pois provoca várias mudanças e fragilidades o que requer uma atenção redobrada por parte dos profissionais de saúde (MARTINS et al., 2011). Na assistencia à saúde da mulher e do recém-nascido o profissional de enfermagem desempenha um papel fundamental, portanto deve promover ações que favoreçam a autonomia da mulher quanto aos cuidados no pré natal, parto, puerpério e nos primeiros cuidados com o recém nascido (COUTINHO et al., 2015).

A Estratégia de Saúde da Família (ESF) ocupa um lugar privilegiado no acompanhamento da mulher durante o pré-natal e puérperio, por ter como princípio a garantia da integralidade das ações e a continuidade do cuidado, e ter seu trabalho pautado no trabalho em equipe, priorização da família em seu território, acolhimento, vínculo, ações de prevenção epromoção da saúde (GARUZ et al., 2014). Na ESF um dos cuidados de enfermagem que deve ser iniciado durante o pré-natal é a promoção do aleitamento materno.

O Ministério de saúde recomenda o aleitamento materno exclusivo até os seis meses de vida, por ser um alimento completo com mais de cento e cinquenta substâncias emsuacomposição que são de extrema importânciapara o crescimento e desenvolvimento saudável da criança (SIMONet al., 2009). A amamentação traz grandes benefícios ao lactente, uma vez que o leite natural possui propriedades nutricionais e imunológicas, protegendo-o de inúmeras doenças e favorecendo seu crescimento e desenvolvimento saudável, além de fortalecer o vínculo mãe-filho e ter efeito protetor nos primeiros meses devida, reduzindo o risco de doenças crônicas autoimunes, alergia alimentar entre outras(MARQUES et al., 2011; CAMPOS et al., 2015).

Avaliando a importância do aleitamento materno para a saúde infantil, a promoção do aleitamento materno deve ser iniciado durante o pré-natal, dessa forma o enfermeiro deve proteger e apoiar esta prática e não deve ter apenas conhecimento empírico e habilidades técnicas, mas arte e sensibilidade para desenvolver na genetriz sentimentos, vontades, induzindo-a ao aleitamento materno (MONTESCHIO et al., 2015). Portanto, o aconselhamento, as informações e a comunicação do enfermeiro constituem-se estratégias no manejo clínico da amamentação. Além disso, o desempenho desse profissional é de grande importância para o apoio e promoção do aleitamento materno exclusivo permitindo sempre buscar maneiras que possam cessar com os medos, frustrações e dificuldades encontradas pelas puérperas no aleitamento dificultando assim, o desmame precoce (AZEVEDO et al.,2015). 
Nesse contexto, avaliar o conhecimento da mãe sobre a importância do aleitamento materno na Atenção Primária, torna-se essencial para que o profissional de saúde possa conhecer os problemas enfrentados por ela durante a amamentação e assim, propor estratégias para solucionar as dificuldades mais comuns encontradas durante esse período.

\section{MATERIAIS E MÉTODOS}

Trata-se de um estudo transversal descritivo de caráter qualitativo, no qual não se preocupou com representatividade numérica, mas em compreender a fundo um grupo de pessoas. A pesquisa qualitativa possibilita que o investigador mergulhe na realidade (ambiente natural) dos indivíduos e produza sobre este uma interpretação que não pode ser quantificada. Leva em consideração crenças, valores e aspectos culturais dos sujeitos da pesquisa (SILVA et al., 2018)

Para realização das entrevistas inicialmente foi realizado um levantamento das mulheres em período puerperal cadastradas na área de abrangência das equipes de saúde da família, com o objetivo de localizar essas pacientes, esse levantamento foi realizada através da coordenação de saúde da família do município de Montes Claros-MG. Posteriormente foi realizado contato com o enfermeiro responsável pela equipe de Saúde da Família na qual as pacientes se encontravam cadastradas, e em seguida foi realizado contato com as pacientes para planejar 0 melhor dia e horário para realização das entrevistas.

Participaram do estudo 12 puérperas por meio de uma entrevista semiestruturadas com utilização de questões norteadoras relacionada ao assunto. Como critérios de inclusão foi utilizado ser puérpera de até 42 dias pós-parto cadastrada na equipe de Saúde da Família e aceitar participar de forma voluntária. Como critérios de exclusão foi utilizado puérpera não encontrada na sua residência após duas tentativas e indicações médicas para substituição parcial ou total do leite materno.

Os dados das entrevistas foram transcritos, categorizados e analisados segundo a análise de conteúdo de Bardin, para melhor compreensão dos sujeitos entrevistados. Para Bardin a análise de conteúdo é uma análise crítica a fim de explorar mais a fundo todos os dados informados.

A análise do conteúdo consiste em três fases, a primeira é uma organização das informações a serem apuradas, a segunda fase consiste na exploração codificação e definição dos conteúdos, e a terceira é a interpretação e tratamento dos dados. (SANTOS, 2015).

A presente pesquisa foi submetida ao Comitê de Ética em Pesquisa das Faculdades Unidas do Norte de Minas (FUNORTE) e autorizada perante o parecer de número 2.570.763. A pesquisa de campo foi realizada no período de abril a maio de dois mil e dezoito, foram realizadas 12 entrevistas e as mesmas foram presenciais, no domicílio de cada paciente, para garantir melhor comodidade e privacidade das mesmas.. Todas as entrevistadas assinaram o Termo de Consentimento Livre e Esclarecido, previamente às entrevistas.

As entrevistas foram gravadas em áudios e posteriormente transcritas para análise. Após transcrição os áudios foram eliminados para garantir sigilo e confidencialidade das informações e das entrevistadas.Com intuito de manter a confidencialidade dos entrevistados, usa-se como forma de identificação das falas, uma letra alfabética ( $A, B, C, D \ldots \ldots L)$, seguida por números identificando a quantidade de falas do entrevistado $(1,2,3 \ldots 12)$.

\section{RESULTADOS E DISCUSSÃO}

As entrevistadas tinham idades que estavam compreendidas na faixa etária de 16 a 37 anos, todas se auto declarava da raça/cor parda, $92 \%$ era casada e/ou união estável e $75 \%$ delas tinham dois ou mais filhos. Em relação a escolaridade $67 \%$ concluiram o ensino médio e $83 \%$ não possuía emprego formal .

Considerando as falas das puérperas sobre o conhecimento do aleitamento materno, foram criadas 04 categorias; "As vantagens do aleitamento materno". "Importância do leite materno sob o olhar da mãe". Informação sobre amamentação no período de pré-natal". "Dificuldades enfrentadas no período de amamentação". 


\section{As vantagens do aleitamento materno}

O aleitamento materno é o método mais natural e seguro de alimentação para os recém-nascidos, devendo ser exclusivo até os 6 meses. A partir dessa idade, deve-se iniciar a complementação com outros alimentos, mantendo o aleitamento materno até, pelo menos, os 2 anos de idade,o aleitamento materno é um ato essencial para o desenvolvimento do recém-nascido, pois o leite da mãe está na temperatura adequada para amamentar e é o único alimento completo e equilibrado que nutre todas as necessidades do bebê (KURTZ et al., 2015).Quando as participantes foram questionadas a respeito do que compreendiam sobre amamentação, identificaram-se nas suas falas diversas informações.

'[...] ele previne muita coisa né? alergia futura e sem contar que o leite é omais forte é mais saudável e ajuda a criança contra alergia ou alguma infecção" (A1)

'[...] É através do leite que ele vai ter proteção, penso na saúde dele"(B1)

'[...] É difícil explicar né? É bom pra criança por que ela não adoece tanto comparada as outras crianças que tomam leite nan"(D1)

"O leite materno é muito importante, porque é o único alimento exclusivo do bebê até o sexto mês" (G1)

"[...] Previne de muitas coisas, de doenças, gripes, infecções e é mais fácil da gente tá alimentando por que ao invés de você estar fervendo mamadeira, comprando leite ele já sai prontinho do peito, na temperatura certa"'(H1)

No relato das puérperas entrevistadas, identificou-se que as mesmas possuem conhecimento sobre os benefícios da amamentação. De acordo com Dodt (2013), o aleitamento materno é ideal para o desenvolvimento psíquico, motor e imunológico do bebê, pois ele é rico em nutrientes e proteínas essenciais para o crescimento da criança. Quase a totalidade da capacidade imunológica é formada durante o aleitamento materno. É nesse momento que enzimas bioativas, hormônios, fatores de crescimento, citocinas e agentes imunológicos agem estimulando e aumentando a defesa do neonato.

\section{Importância do leite materno sob o olhar da mãe}

O aleitamento materno deve ser visto sob a óptica da mulher no sentido de compreender suas reais necessidades, concepções e dificuldades, enquanto profissionais de saúde, deve-se realizar uma escuta ativa, adaptar a assistência através de uma equipe interdisciplinar, fornecendo apoio adequado e esclarecendo crenças e tabus(MOURA et al., 2015).

"[...] Eu acho que pro bebê é essencial o leite materno, tanto que é o segundo filho, o primeiro eu amamentei até 1 ano e 3 meses e eu acho que ajuda também no sentido de ligação entre a mãe e filho"'(A2)

"[...] A importância do leite materno é a saúde do meu bebê, penso que é através do leite que ajuda no desenvolvimento dele, acho que é só isso" (C1)

"[...] É a base de tudo né? A base de tudo da boa saúde do bebê é o aleitamento materno" (E1)

'[...] É a segunda gestação que eu tenho, a primeira graças a Deus foi excelente, agora o segundo filho está sendo a mesma coisa é importante a amamentação porque é o melhor alimento pra criança né? " (D2)

"[...] É importante porque é através dele que protege o bebê contra doenças, gripes, muitas coisas é bom demais" (F1)

'[...] ...é importante porque a criança fica saudável né?"(H2) 
"Fortalece o vínculo entre eu e meu neném, pelo que eu sei o organismo dele ainda não está amadurecido para outro tipo de alimentação então vou oferecer o leite materno até os sexto mês, é bom também para nutrição porque com o leite materno ele fica mais gordinho".(J1) '(I1)

'[...] Ajuda no desenvolvimento do bebê, o sistema imunológico dele fica mais resistente a infecções

O entendimento das puérperas sobre o aleitamento materno influencia diretamente na suas atitudes em relação ao ato de amamentar. Sendo possível verificar um conhecimento espontâneo unindo a amamentação à prevenção de doenças e fortalecimento do vínculo mãe/filho.O aleitamento materno tem sido considerado estratégia natural de vínculo, afeto, proteção e nutrição para a criança, sendo uma intervenção eficaz para a redução da morbimortalidade infantil (ORSOet al., 2016).

Segundo Passanha et al. (2013), o aleitamento materno é a melhor forma de alimentação das crianças recém-nascidas e mostra grandes benefícios quando continuada até os dois anos de idade, promove benefícios e desenvolvimento integral para a saúde da criança, nenhum outro alimento é capaz de oferecer ao lactente o que há no leite materno. Além de favorecer o contato mais íntimo entre mãe e filho, o leite materno apresenta substâncias que se ajustam normalmente às necessidades nutricionais dos lactentes.

\section{Informação sobre amamentação no período de pré-natal}

De acordo com Ferreira et al. (2018), as infomações repassadas pelos profissionais de saúde influenciam diretamente na alimentação, nutrição e prevenção de doenças na vida da criança. É importante que a mãe seja orientada em relação ao aleitamento materno desde a primeira consulta de pré-natal.Dessa forma, a equipe multiprofissional deve incentivare informar as mães sobre a importância do aleitamento materno, as vantagens e as dificuldades vivenciadas pela mulher durante essas consultas.

'[...] nesse sentido específico não. Já tinha um pouco de conhecimento por que é meu terceiro filho eu questionei sobre o bico invertido o que fazer por que eu tenho bico invertido, o enfermeiro disse que eu não poderia estimular o bico no sentido de puxar para fora porque poderia sair o leite e no sentido de banho de sol e bucha vegetal poderia ferir o peito" (A3)

'[...] Foi falado lá no pré-natal que o leite materno previne muitas coisas, alergias futuras e que sem contar que o leite é o mais forte é mais saudável que ajuda combater alergias e alguma infecção" (B3)

"[...] Foram dadas várias orientações, mais a que recordo é que ajuda no crescimento e desenvolvimento do bebê e que é bom o uso exclusivo até o sexto mês de vida" (G2)

'[...] A informação que eu tenho que é bom porque previne doenças (K1)

"[...] Me falaram que aumenta o vínculo entre eu meu filho, melhora a imunidade dele e também amamentando ajuda o meu corpo a voltar mais rápido e também o útero voltar mais rápido para o lugar" (I2)

'[...] Me informaram que a mamada tem que ser mais longa em cada mama por que o primeiro leite é matar a sede o segundo é para nutrir e o terceiro é o que temgordura que é pra ele engordar, fortalecer" (J2)

O profissional de saúde, em especial o enfermeiro devem incentivar o aleitamento materno por estar mais próximo do binômio mãe-filho. Durante a assistência ao pré-natal e no período do puerpério,o profissional deve certificar-se quanto aos conhecimentos da paciente, sendo ele de caráter intelectual ou cultural (CAMPOSet al., 2015).Entretanto, houve puérperas que relataram não ter recebido informações, conforme se constata nas narrativas:

'[...]...não tive nenhuma orientação, era muito bagunçado, marca consulta lá acho que tive umas 4 consultas, mas tive uma dúvida, perguntei a enfermeira se o meu peito rachasse o que eu passar, ela me respondeu que deveria fica no sol e passa bucha vegetal. "(D3)

"[...] ...não, não recebi..." (C2) 


\section{"[...] Não, não tive nenhuma informação, lá apenas no hospital." (E2)}

A educação em saúde, durante a gestação e após o nascimento dos bebês, é importante para a promoção de aleitamento materno,o acompanhamento das mães durante a lactação é fundamental, mostrando a importância de não só informar, mas também apoiar e acompanhar essas mulheres para que se tenha sucesso na prática do aleitamento materno (MOIMAZet al.,2013).

Observa-se que o profissional de saúde tem um papel importante frente às dúvidas e orientações que surgem durante o pré-natal, no incentivo à amamentação e em todas as fases do pré-natal e puerpério,através das visitas domiciliares, palestras, grupos de gestantes e a manutenção do período puerperal.Porém, estudos apontam falhas no processo de orientação com relação ao pré-natal, tanto nos aspectos teóricos como atividades exclusivamente práticas (SILVIA et al., 2014).

\section{Dificuldades enfrentadas no periodo de amamentação}

As dificuldades na amamentação não se apresentam de forma isolada, mas sim interligadas em um conjunto de fatores, sustentando assim a importância do acompanhamento profissional para que desta forma o aleitamento materno não seja interrompido. É necessário acompanhar as mães no período da lactação, identificando precocemente dificuldades que podem vir a surgir, porque se esses problemas não forem bem acompanhados e assistidos podem acarretar a interrupção precoce do aleitamento (VARGAS et al., 2016 ; MOIMAZet al., 2013).Os relatos abaixo são experiências difíceis e dolorosas vivenciadas pelas mulheres durante oprocesso de lactação:

"[...] eu tenho bico invertido e isso me traz bastante dificuldade." (A4)

"[...] Apesar de ser a terceira filha tive dificuldade de amamentar no começo pois ela não sugava adequadamente, feriu demais e sangrava muito meu peito, mais mesmo assim eu não desisti e depois de uns dias ela começou a pegar certinho, acho que se no meu caso eu não tivesse insistido, hoje não estaria mais amamentando." $(\mathrm{H} 2)$

"[...] No início tive muita dificuldade que foi a demora da descida do leite, tive que passar por procedimento de massagem, tomar medicamentos para ver se aumentava o leite, pois não estava saindo nada." (I2)

'[...] Minha maior dificuldade foi referente a pega, foi muito difícil pois no começo descia pouco leite e o bebê ficava agitado, e também amamentar de madrugada a gente fica muito cansada." (J3)

"[...] Tive dificuldade porque meu peito rachou e não sabia o que fazer aí com o próprio leite materno eu passo no bico do peito e fico no sol, por que disseram que não pode passa pomada mais dói muito quando ela mama." (F3)

Dificuldades relacionadas às mamas caracterizaram a principal dificuldade com a técnica da mamada, o que pode fazer com que a criança seja amamentada menos vezes. Tais dificuldades podem ocorrer devido à falta de acesso às orientações, apoio social e profissional durante a gestação e no pós-parto. É necessário que as mulheres que vivenciaram um processo de amamentação traumático sejam assistidas mais de perto pelos profissionais de saúde, que devem orientar a técnica correta (AMARAL et al., 2015).

De acordo com Almeida et al. (2015), alguns profissionais são poucos capacitados sobre a importância da amamentação, demonstrando um baixo manejo de habilidade e poder deconversação entre ele e a mãe. Cabe ressaltar que deve ser prioritária a implantação de estratégias políticas favorecendo o incentivo ao aleitamento materno.

\section{CONCLUSÃO}

Tendo em vista que o aleitamento materno é indiscutivelmente o melhor alimento a ser ofertado até o sexto mês de vida, pois oferece todos os nutrientes que o bebê precisa, os profissionais devem oferecer apoio, realizar uma escuta ativa, identificar demandas de cuidado, auxiliar na implementação de medidas para auxiliar o 
processo de amamentar e, além disto, devem estar preparados para influenciar significativamente a prática do aleitamento. Percebe-se pela pesquisa que existe um conhecimento prévio das puérperas sobre 0 aleitamento no entanto , parte dos profissionais de saúde necessitam abordar mais o tema durante o acompanhamento ao pré-natal e puerpério. Além da oferta de ações de educação em saúde para esclarecimento de dúvidas, crenças e mitos acerca da amamentação e assim colaborar para diminuiçãodo índice de desmame precoce.

\section{REFERÊNCIAS}

1. AMARAL LJX, SALES SS, CARVALHO DPSRP et al. Fatores que influenciam na interrupção do aleitamento materno exclusivo em nutrizes.Rev Gaúcha Enferm, 2015.

2. ALMEIDA JM, LUZ SAR, VEIGA UEDF. Apoio ao aleitamento materno pelos profissionais de saúde: revisão integrativa da literatura. Revista Paulista de Pediatria, 2015.

3. AZEVEDO ARR, ALVES VH, SOUZA RMP et al. Manejo clínico de amamentação. Escola Anna Nery Revista de enfermagem.2015.

4. CAMPOS AMS, CHAOUL CO, CARMONA EV et al .Prática do aleitamento materno exclusivo informada pela mãe e oferta de líquidos aos seus filhos. Revista Latino Americana de Enfermagem, 2015.

5. COUTINHO LRP,BARBIERI AR, SANTOS MLM. Acolhimento na Atenção Primária à Saúde: Revisão integrativa. Revista Saúde Debate, 2015.

6. DODT RCM, FERREIRA AMV, NASCIMENTO LA, et al Influence of health education strategy mediated by a self-efficacy breastfeeding serial album. Texto \& contexto enferm, 2013.

7. FERREIRA MGC, GOMES MFP, FRACOLLI LA. Aleitamento materno: Orientações recebidas por gestantes acompanhadas pela estratégia da saúde da família. Rev. Aten.Saúde,São Caetano do Sul, 2018.

8. GARUZI M, ACHITTI MCO, SATO CA et al. Acolhimento na Estratégia Saúde da Família: Revisão integrativa. Revista Panamericana de Salud pública, 2014.

9. MARTINS AB, RIBEIRO J, SOLER ASG.Proposta de exercícios físicos no pós -parto: um enfoque na atuação do enfermeiro obstetra. Invest. Educ Enferm,2011.

10. MARQUES ES , COTTA RMM, PRIORE SE. Mitos e crenças sobre o aleitamento materno. Revista Ciência \& Saúde Coletiva, 2011.

11. MEDEIROS LS, COSTA ACM. Período puerperal: a importância da visita domiciliar para enfermeiros da Atenção Primária à Saúde. Rev Rene. 2016

12. MOIMAZ SAS, SALIBA O, BORGES HC et al. Desmame Precoce: Falta de Conhecimento ou de Acompanhamento. Pesq Bras Odontoped Clin Integr, 2013.

13. MOURA ERBB ,FLORENTINO ECL, BEZERRA MEB . Investigação dos fatores sociais que interferem na duração do aleitamento materno exclusivo. Revista Intertox-EcoAdvisor de Toxicologia Risco Ambiental e Sociedade, 2015.

14. MONTESCHIO CAC, GAIVA MAM, MOREIRA MDS. The nurse faced with early weaning in child nursing consultations. Rev Bras Enferm.2015.

15. ORSO LF, MAZZETTO FMC, SIQUEIRA FPC. Percepção de mulheres quanto aos cenários de cuidado em saúde na promoção do aleitamento materno.Revista Recien, 2016.

16. PASSANHA A, AQUINO BMA, ISOYAMA VS et al. Implantação da rede amamenta Brasil e prevalência de aleitamento materno exclusivo. Revista de Saúde Pública, 2013.

17. RAMOS LC. Aleitamento Materno- efeito de intervenção educativa com equipe de enfermagem na orientação a nutrizes.2014.

18. SANTOS ACC. Relato de experiência no contexto de educação em saúde o cuidado, materno- infantil. 2015.

19. SILVA NM, WATERKEMPER R, EVELINE FS et al., Conhecimento de puérperas sobre amamentação exclusiva. Revista Brasileira de Enfermagem,2014.

20. SILVA RM et al (Orgs).Estudos Qualitativos:enfoques teóricos e técnicas de coleta de informações. Sobral: edições UVA;2018;305p

21. SIMON VGN,SOUZA JMP, SOUZA SB. Aleitamento materno, alimentação complementar, sobrepeso e obesidade em pré-escolares. Rev. Saúde Pública,2009.

22. KURTZ L, MAAHS MAP, BONAMIGO AW et al. Promoção do aleitamento materno em um contexto interdisciplinar. Rev. de atenção à saúde, 2015.

23. VARGAS GSA, ALVES VH, RODRIGUES DP et al. Atuação dos profissionais de saúde da estratégia saúde da família: promoção da prática do aleitamento materno. Revista Baiana de Enfermagem,2016. 